Revue

de l'histoire des religions
Revue de l'histoire des religions

$4 \mid 2014$

L'objet rituel. Concepts et méthodes croisés

\title{
Les objets rituels des Juifs à la fin du Moyen Âge, catalyseurs des sentiments antijuifs ou fédérateurs des identités?
}

Jewish ritual objects in the late Middle Ages: Catalyzing anti-Jewish sentiments or unifying identities?

\section{Claire Soussen}

\section{OpenEdition}

Journals

Édition électronique

URL : http://journals.openedition.org/rhr/8326

DOI : $10.4000 /$ rhr.8326

ISSN : 2105-2573

Éditeur

Armand Colin

Édition imprimée

Date de publication : 1 décembre 2014

Pagination : 681-698

ISSN : 0035-1423

Référence électronique

Claire Soussen, « Les objets rituels des Juifs à la fin du Moyen Âge, catalyseurs des sentiments antijuifs ou fédérateurs des identités? ", Revue de l'histoire des religions [En ligne], 4 | 2014, mis en ligne le 01 décembre 2017, consulté le 19 avril 2019. URL : http://journals.openedition.org/rhr/8326 ; DOI : $10.4000 /$ rhr.8326 


\section{Les objets rituels des Juifs à la fin du Moyen Âge, catalyseurs des sentiments antijuifs ou fédérateurs des identités?}

Après s'être interrogé sur la notion d'objet rituel et sa validité pour l'époque médiévale, l'article s'attache à examiner les différents objets qui apparaissent comme symboliques des Juifs (kippah, franges rituelles, couteau de la circoncision ou de l'abattage rituel), mais aussi des objets non «identitaires» et la façon dont ils sont appréhendés dans le cadre des relations entre Juifs et Chrétiens à la fin du Moyen Âge dans l'espace ibérique. Utilisés dans le cadre de rites spécifiquement juifs ou dans des rituels exogènes, ils apparaissent comme des objets transitionnels dans l'expression des sentiments, positifs ou négatifs, des groupes et des individus. L'iconographie et les sources textuelles (documents normatifs et de la pratique, traités de théologie et commentaires bibliques et talmudiques) éclaireront l'analyse.

\section{Jewish ritual objects in the late Middle Ages : Catalyzing anti-Jewish sentiments or unifying identities?}

After questioning the notion of ritual object and its validity for the Middle Ages, the article will examine the different objects which seem to symbolize the Jewish identity through the ages (kippah, ritual fringes, circumcision and ritual slaughter knife), and also objects without religious symbolism and the way they are considered within the relations between Jews and Christians in the late Middle Ages in the Iberian area. Used for specifically Jewish rites or within profane rites, they act as transitional objects to express positive or negative feelings, by groups or individuals. Iconography and textual documents (expressing the norm or reflecting the practical field, theological treatises and biblical or talmudical commentaries) will support the analysis. 
Contrairement à ce que le titre - résolument affirmatif - de cet article pourrait faire croire, le concept d'objet rituel ne va pas de soi pour le Moyen Âge. Alors que le non spécialiste de l'histoire de l'Antiquité pourrait penser que la religion des Grecs en regorge, notamment sous la forme des offrandes - mais les offrandes sontelles des objets rituels ou des objets manipulés dans un contexte rituel ? -, le médiéviste a du mal à proposer une définition, ou retenir une acception précise de l'objet rituel. Alors que l'historiographie allemande en particulier ${ }^{1}$, et de façon pionnière, a pris les rituels médiévaux pour thème d'étude privilégié et qu'elle a entraîné dans son sillage les travaux des historiens français, certaines critiques se sont élevées contre la tendance à voir du rituel dans tout ou du moins à considérer comme rituels des faits ou des pratiques qui ne l'étaient pas pour leurs contemporains'2.

De fait, le concept d'objet rituel n'apparait pas dans la documentation médiévale, il est un produit construit par les historiens qui l'analysent comme support matériel de cérémoniaux divers. Pour aider à trouver l'objet rituel au Moyen Âge, il faut peut-être évoquer une autre catégorie d'objets qui existent de manière incontestable, les objets liturgiques, et s'interroger sur ce qui les distingue d'objets que l'on qualifierait de rituels. À la première catégorie ressortissent de nombreux objets manipulés dans le cadre liturgique, ecclésial ou synagogal: on pense entre autres aux encensoirs, crucifix, missels et autres livres liturgiques, couronnes et rouleaux, mais aussi aux reliquaires ou aux statues portées en procession ${ }^{3}$. À la seconde catégorie ressortissent des objets qui ne sont pas rituels stricto sensu, mais qui le deviennent par l'usage qui en est fait: on pense au chien porté sur le cou des condamnés au rituel de l'amende honorable étudié magistralement par Jean-Marie Moeglin4, ou aux

1. Voir notamment les travaux de Gerd Althoff, Die Macht der Rituale, Darmstadt, Primus Verlag, 2003.

2. Philippe Buc, Dangers of Ritual, Princeton, Princeton University Press, 2001, trad. fr.: Dangereux rituels - de l'histoire médiévale aux sciences sociales, Paris, PUF, 2003.

3. Voir entre autres Éric Palazzo, L'espace rituel et le sacré dans le christianisme. La liturgie de l'autel portatif dans l'Antiquité et au Moyen Âge, Turnhout, Brépols, 2008.

4. Jean-Marie Moeglin, «Harmiscara/ Harmschar/ Hachée. Le dossier des rituels d'humiliation et de soumission au Moyen Âge», Archivum Latinitatis 
deniers posés sur la tête des serfs qui reconnaissent de la sorte leur dépendance. Cela dit, à mesure que l'on avance dans le Moyen Âge, à une époque où la société s'est profondément christianisée et où le facteur religieux est englobant, peut-il y avoir des objets rituels non liturgiques? Même lorsqu'on pense spontanément aux objets qui apparaissent de façon récurrente dans les cérémonies d'hommage vassalique, fétu de paille, motte de terre, symbolisant la contrepartie donnée au vassal par son seigneur en échange de

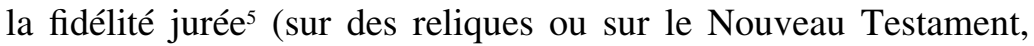
pleinement objets rituels dans ce cas), il apparaît qu'avec le temps, et la grande diffusion de la féodalité, la cérémonie elle-même s'est profondément christianisée et que le rituel qui l'entoure s'apparente de plus en plus à une forme de liturgie. Il en va de même si l'on pense aux regalia, ces objets - sceptre, couronne, éperons, épée qui symbolisent la royauté et qui n'ont aucune valeur religieuse intrinsèque, mais qui sont auréolés d'une dimension sacrée et manipulés dans des rituels profondément religieux, le sacre des rois de France en étant l'exemple le plus éclatant ${ }^{6}$. Les objets purement et seulement rituels, c'est-à-dire manipulés dans un contexte non liturgique et sans valeur liturgico-religieuse intrinsèque, semblent alors de moins en moins faciles à appréhender, même s'il continue d'en exister.

Par ailleurs, il semble que la quête de l'objet rituel médiéval ait tout à gagner à sortir du cadre exclusivement chrétien. Les relations entre Juifs et Chrétiens à la fin du Moyen Âge, thème qui polarise ma recherche, donnent à voir un certain nombre d'objets manipulés

Medii Aevi, Bulletin Du Cange, t. LIV, 1996, p. 11-65. Voir aussi J.-M. Moeglin, «Le Christ la corde au cou», La dérision au Moyen Âge. De la pratique sociale au rituel politique, dir. Élisabeth Crouzet-Pavan, Jacques Verger, Paris, PUPS, 2007, p. 275-289. Dans cet article, J.-M. Moeglin livre une nouvelle lecture du rituel de l'Harmiscara, qui n'apparaît plus déconnecté de la sphère liturgico-religieuse.

5. Gerd Althoff et Christiane Witthöft, «Les services symboliques entre dignité et contrainte», Annales. Histoire, Sciences Sociales, 2003/6, 58 année, p. 1293-1318, p. 1294. Voir aussi Hélène Débax, «Le serrement des mains. Éléments pour une analyse du rituel des serments féodaux en Languedoc et en Provence ( $\mathrm{XI}^{\mathrm{e}}-\mathrm{XII}^{\mathrm{e}}$ siècles)», Le Moyen Âge, 2007/1, t. CXIII, p. 9-23.

6. Jacques Le Goff, Éric Palazzo, Jean-Claude Bonne, Marie-Noël Colette, Le Sacre royal à l'époque de saint Louis d'après le manuscrit latin 1246 de la $B n F$, Paris, Gallimard, 2001 ; J.-M. Moeglin, «Rituels et "Verfassungsgeschichte" au Moyen Age. À propos du livre de Gerd Althoff, Spielregeln der Politik im Mittelalter-Kommunikation in Frieden und Fehde», Francia, t. 25, 1998, p. 245250 . 
dans un cadre rituel qu'il convient d'analyser dans leurs contextes afin de déterminer leur nature et caractériser leurs fonctions. Pour cela il faut accepter d'envisager non seulement les objets rituels définis stricto sensu, c'est-à-dire en l'occurrence dont la forme ou la nature ressortissent exclusivement aux rites juifs, mais aussi d'autres objets qui, par l'usage qu'on en fait, deviennent de facto des objets rituels. Par ailleurs, si l'on veut embrasser un champ de réflexion vraiment large qui permette d'ouvrir et d'enrichir le questionnement, on examinera aussi des objets qui, sans avoir la moindre coloration rituelle, prennent un sens particulier lorsque le contexte de leur utilisation en vient à leur donner une dimension rituelle.

C'est en ayant toutes ces questions à l'esprit que j'ai choisi d'observer la façon dont les objets rituels des Juifs sont appréhendés par les Juifs et les non-Juifs dans le contexte spécifique de l'espace aragonais à la fin du Moyen Âge. Comment, à travers ces objets d'emblée rituels ou au contraire résultats d'une projection de la part de ceux qui les manipulent ou les voient manipulés, se traduisent la richesse et les enjeux des relations entre Juifs et Chrétiens à un moment de tension historique? J'observerai donc tour à tour les objets rituels des Juifs, invariants et fédérateurs, dans un deuxième temps les objets et symboles des Juifs dans des rituels nonjuifs, et enfin les objets associés aux rites juifs comme agents de cristallisation des sentiments antijuifs.

\section{LES OBJETS RITUELS DES JUIFS, INSTRUMENTS FÉDÉRATEURS}

\section{Les objets rituels identitaires : tsitsit, tefillin, kippah}

Un certain nombre d'objets associés au rite, à la liturgie ou au mode de vie juifs en sont venus, avec le temps et la diversité de leurs contextes d'utilisation, à acquérir une dimension identitaire. Dans la diaspora, leur pouvoir identificateur est encore accru car ils agissent comme des instruments fédérateurs, des symboles identitaires. Ces objets symboliques, tsitsit, tefillin, kippah, trouvent pour certains leur origine dans la Bible, d'autres dans le Talmud. Nous pouvons qualifier ces objets d'objets rituels identitaires car ils signent immédiatement et exclusivement la judéité de ceux qui les portent (à distinguer d'autres objets rituels utilisés par les Juifs de manière 
spécifique, mais qui ne sont pas «juifs» par nature). Les premiers de ces objets sont sans doute les tsitsit, ou franges rituelles, dont la prescription figure dans le Pentateuque, Nb. 15. 37-38: «L'Éternel parla à Moïse en ces termes: "Parle aux enfants d'Isräl, et dis-leur de se faire des franges aux coins de leurs vêtements dans toutes leurs générations et d'ajouter à la frange de chaque coin un cordon d'azur" " ${ }^{7}$. Ces franges rituelles doivent donc être portées tous les jours et pour toujours. Elles le sont sous la forme du châle de prière, porté en quelques moments précis de la journée, ou sous la forme du petit tallit, porté sous les vêtements, à longueur de journée. Pour le Moyen Âge, si l'iconographie ne retient pas les franges rituelles comme thème privilégié d'identification des Juifs, nous trouvons pourtant la mention de cet objet rituel dans la documentation textuelle. En effet, en 1215, le canon 68 du $4^{\text {e }}$ concile de Latran au cours duquel est proclamée la nécessité du port de signes distinctifs (dont la forme n'est pas précisée à ce stade) par les Juifs et les Musulmans, fournit la justification de cette mesure: afin que les Chrétiens, les Juifs et les Musulmans se distinguent les uns des autres ${ }^{8}$. Par ailleurs, le canoniste ajoute une justification à cette mesure: "Il leur est aussi demandé de le faire (sous-entendu se distinguer) par Moïse $»^{9}$, ce qui renvoie explicitement à Nb. 15-38. Le caractère identitaire des franges rituelles est donc perçu par tous, non-Juifs compris, même si le rappel de la nécessité de les porter n'est évidemment pas motivé par les mêmes intentions. Cela dit, ce que l'on peut déduire de ces citations, c'est que les franges rituelles n'étaient en réalité pas portées, puisque le canon explique que les Juifs ne se distinguent pas des Chrétiens.

7. Bible du Rabbinat. Le texte se poursuit et justifie la prescription en $\mathrm{Nb}$. 15. 39: «Cela formera pour vous des franges dont la vue vous rappellera tous les commandements de l'Éternel, afin que vous les exécutiez et ne vous égariez pas à la suite de votre cœur et de vos yeux, qui vous entraînent à l'infidélité».

8. Quatre canons, les canons 67, 68, 69, 70 du $4^{\text {e }}$ Concile de Latran promulgués le 11 novembre 1215 et édités dans Mansi, Sacrorum Conciliorum nova et amplissima collectio, Paris-Florence, t. 22, évoquent la question des relations avec les Chrétiens. Le titre du canon 68 est explicite: «Ut Judei discernantur a Christianis in habitu» et le texte développe: «In nonnullis provinciis a christianis iudeos seu sarracenos habitus distinguit diversitas, sed in quibusdam sic quaedam inolevit confusio ut nulla differencia discernantur. Unde contingit interdum quod per errorem christiani iudeorum seu sarracenorum et iudei seu sarraceni christianorum mulieribus commisceantur ».

9. Ibid.: «cum etiam per Mosen hoc ipsum legatur eis injunctum». 
Cela nous conduit à porter le questionnement sur un autre objet identitaire (endogène ou exogène, la question se pose): le couvrechef, kippah, chapeau qui lui, en revanche, est bien représenté dans l'iconographie. Le thème du chapeau juif, ou chapeau des Juifs, apparaît en effet fréquemment dans les manuscrits de la sphère ashkénaze $^{10}$, mais qu'en est-il réellement de son usage, est-il un objet rituel au sens propre, c'est-à-dire ressortissant uniquement au rite juif? Pour ce qui est de son origine, contrairement à ce que l'on pense parfois, elle n'est pas biblique ni même talmudique. La Bible, en Exode 28.4, prescrit le port d'un couvre-chef uniquement pour les prêtres, les Cohanim, pour lesquels il représente une marque de prestige à l'instar d'une couronne ${ }^{11}$. Ezéchiel 24. 23 ajoute: "Votre coiffure restera sur votre tête et vos chaussures à vos pieds». À l'époque talmudique, le port d'un couvre-chef se généralise pour les hommes pieux en signe de révérence envers Dieu. Le traité Kidouchin 31a enseigne que la présence divine se trouve au-dessus des hommes; et ceux-ci se couvrent la tête pour montrer qu'ils en sont constamment conscients ${ }^{12}$. C'est au $\mathrm{XVI}^{\mathrm{e}}$ siècle que le Choul'hane Aroukh, ouvrage de casuistique et guide de pratiques rédigé par Joseph Caro, prescrit le port de la kippah pour tous et tout le temps et précise que nul homme ne peut marcher tête nue sur une distance dépassant quatre coudées ${ }^{13}$, reprenant ainsi la recommandation talmudique. Les polémistes juifs justifient d'ailleurs parfois cette obligation par la volonté assumée des Juifs de se distinguer des Chrétiens qui, eux, se défont de leur couvre-chef lorsqu'ils entrent dans un lieu consacré. La kippah n'est donc pas, au départ, un objet rituel; elle l'est devenue petit à petit, même si dès le Moyen Âge central, l'iconographie chrétienne représente les Juifs coiffés.

Enfin, il est un objet rituel des Juifs qui n'est pas mentionné dans la documentation chrétienne, et qui est à la fois rituel et liturgique:

10. Bernhard Blumenkranz, Le juif médiéval au miroir de l'art chrétien, Paris («Études Augustiniennes, $2 »), 1966$, p. 46-47.

11. Exode 28. 4: «Or voici les vêtements qu'ils exécuteront: un pectoral, un ephod, une robe, une tunique à mailles, une tiare et une écharpe; ils composeront ainsi un saint costume à Aaron ton frère et à ses fils, comme exerçant mon ministère».

12. Talmud, traité Kidouchin 31a: «Rabbi Huna fils de Rabbi Joshua n'aurait pas marché tête nue sur plus de quatre coudées car il disait: "la Shekhinah est au-dessus de ma tête"».

13. Chlomoh Ganzfried, Abrégé du Choul'hane Aroukh, Paris, Colbo, 1993, p. 11. 
il s'agit des phylactères dont le port sur le front et le bras gauche est prescrit par la Bible, Deutéronome 6. $8^{14}$. On sait l'importance des tefillin pour les Juifs de toutes les époques car ils sont mentionnés par les Halakhistes, auteurs de responsa (ces questions adressées par tout type d'individus ou représentants des communautés aux autorités religieuses de leur temps), de manière récurrente. Rachi notamment, au XI ${ }^{\mathrm{e}}$ siècle, prescrit des modalités spécifiques pour les attacher. À la fois objet liturgique et rituel de par la complexité et la précision des gestes accomplis pour les poser, ils ne semblent pas attirer l'attention des Chrétiens. Objets spécifiquement juifs par leur forme et par leur sens car contenant sur un petit bout de parchemin le texte des principaux commandements donnés par Dieu à Moïse, ils sont symboliques de l'identité juive.

\section{Les objets rituels non identitaires, mais garants de l'identité juive}

Il s'agit en réalité d'un même objet, mais dans deux contextes d'utilisation différents. Les couteaux, qui n'ont au départ aucun caractère juif, mais par les usages qui en sont faits constituent de vrais objets rituels du judaïsme: le couteau du mohel, le circonciseur, et le couteau du shohet, l'abatteur rituel. Le premier consacre l'entrée dans la communauté des garçons nouveau-nés ${ }^{15}$ et commémore ce que l'on a coutume d'appeler le sacrifice d'Abraham, c'est-à-dire la preuve de l'abandon total d'Abraham à Dieu, qui comprend l'offrande de son fils en signe de dévotion ${ }^{16}$. L'ablation du prépuce des garçons, prescrit par Lv. 12. $3^{17}$, rappelle le don de la vie d'Isaac finalement sauvé par Dieu. Le traité du Talmud Shabbat 130-134, expose les développements nécessaires à l'accomplissement du

14. Deutéronome 6. 8: «Tu les attacheras comme symbole sur ton bras et les porteras en fronteau entre tes yeux».

15. Patricia Hidiroglou, Les rites de naissance dans le judaïsme, Paris, Les Belles Lettres, 1997, p. 107 et suivantes. Voir aussi Nicole Belmont, «La notion de rite de passage », Les rites de passage aujourd'hui. Actes du colloque de Neuchâtel, 1981, dir. Pierre Centlivres, Jacques Hainard, Lausanne, l'Âge d'homme, 1986, p. 9-19.

16. Gn. 22.2: «Il reprit: "Or çà, prends ton fils, ton fils unique, celui que tu aimes, Isaac; achemine-toi vers la terre de Moria, et là offre-le en holocauste sur une montagne que je te désignerai" ».

17. Lv. 12. 3: «Au huitième jour, on circoncira l'excroissance de l'enfant». Prescrit déjà en Gn. 17. 14: «Et un mâle incirconcis qui ne circoncira pas la chair de son prépuce, cette âme sera retranchée de son peuple.» 
rituel ${ }^{18}$. Dans les deux cas, c'est par un couteau que le geste est accompli, le rite produit. Pour ce qui est de la forme du couteau, de ses caractéristiques matérielles, les précisions sont rares. Si le sage cordouan Maïmonide, à la fin du XII ${ }^{\mathrm{e}}$ siècle, donne dans son Mishne Torah, ouvrage halakhique, de nombreuses précisions quant aux règles à suivre lors de la circoncision, il ne décrit pas précisément l'objet. L'iconographie médiévale, qui représente assez fréquemment la scène du sacrifice d'Abraham dans les Bibles historiées, figure le plus souvent le couteau sous la forme d'une épée. On voit ainsi Abraham attraper Isaac par les cheveux et prêt à lui trancher la tête dans une Bible réalisée vers 1330 et conservée à la Bibliothèque Sainte-Geneviève ${ }^{19}$. Les miniatures représentant la circoncision du Christ figurent un couteau, et montrent l'enfant Jésus dénudé et sur le point d'être circoncis ${ }^{20}$. Quant aux textes des polémistes chrétiens, ils n'évoquent pas le détail du rite puisqu'ils le dénoncent comme obsolète. Ainsi Bernard Oliver dans son Contra cecitatem Iudeorum rédigé à la fin du XIII siècle, considère que l'Ancienne Loi - c'est-àdire la Loi de l'alliance - ayant cessé, le signe distinctif que constituait la circoncision et qui distinguait les Juifs adeptes de cette loi doit être aboli. De ce fait donc, la circoncision n'a plus lieu d'être ${ }^{21}$.

Mais pour les Juifs, la circoncision est un rite majeur, un rite fondateur. Rite de passage, rite de consécration, geste de dévotion, la circoncision est tout cela à la fois. Outil du rite, le couteau de la circoncision, même s'il n'est pas en soi un objet spécifique, est bien un objet rituel.

18. Il insiste notamment sur la prééminence de la circoncision parmi tous les rites du judaïsme et notamment par rapport au shabbath. Il évoque ensuite les gestes rituels à accomplir pour le bon déroulement du rite et la nécessité de tenir compte de l'état de l'enfant avant de le pratiquer, même si la règle est de l'accomplir le huitième jour après la naissance. Maïmonide dans le $2^{\mathrm{e}}$ livre de son code halakhique, le Mishne Torah, développe les diverses procédures à suivre; voir Moïse Maïmonide, The Code, Book 2: The book of love, trad. Menachem Kellner, New Haven, Yale University Press, 2004.

19. Exemple d'une Bible historiée, vers 1330, Paris, Bibl. Sainte-Geneviève, ms. $22, f^{\circ} 27$.

20. Exemple d'un missel réalisé vers 1460 et conservé à la Bibliothèque municipale de Clermont-Ferrand.

21. Bernard Oliver, Contra cecitatem Iudeorum, éd. Francisco Cantera Burgos, Madrid-Barcelone, 1965. Chapitre 6, p. 116: «Si vero loquaris de circumcisione carnali tunc accipe sempiternum secundo modo dictum quia quamdiu lex duravit que solum uni populo precipiebatur debuit durare signum quo ille populus ab aliis gentibus distingebatur et lege cessante, eciam signum debuit cessare». 
Il existe un deuxième couteau, tout aussi important, cette fois pour la pratique quotidienne des Juifs, le couteau du shohet dit halef, qui fait l'objet de dispositions particulières précisées dans le traité Houlin 17b du Talmud de Babylone. Il s'agit d'un couteau en acier, dont la longueur égale deux fois la largeur du cou de l'animal, ce qui permet de pratiquer l'incision sans interruption; sa section est presque triangulaire; son extrémité est rectiligne, perpendiculaire à son axe, jamais pointue, pour ne pas risquer de perforer au lieu d'inciser. Comme la circoncision, l'abattage rituel fait l'objet de questionnements récurrents au fil des siècles, et les grands décisionnaires juifs écrivent beaucoup à ce sujet. Maïmonide, toujours dans son Michne Torah ${ }^{22}$, précise que s'il y a un sillon, même très petit, sur le fil du couteau, l'abattage est impropre. Et dans la deuxième moitié du $\mathrm{XVI}^{\mathrm{e}}$ siècle, le Choul'hane Aroukh précise qu'avant chaque saignée, il faut faire l'épreuve du couteau sur le fil et sur chaque face du fil en passant l'ongle et la pulpe du doigt (Yoré Déa 18, 9) ${ }^{23}$. En théorie donc, les Juifs ne peuvent consommer de viande que si elle est abattue rituellement. Les gestes particuliers qui président à l'abattage, le soin avec lequel le shohet examine le couteau, font de l'ensemble du rituel une procédure qui garantit la spécificité du groupe, préserve et pérennise son identité.

Examinons à présent les objets ou supports à partir desquels sont élaborés des rituels non-juifs mais associés aux Juifs.

\section{LES RITUELS NON-JUIFS À PARTIR D'OBJETS DU RITE JUIF}

La documentation de la pratique, qu'il s'agisse de recueils de coutumes ou de documents de la chancellerie royale aragonaise, révèle plusieurs procédures ou cérémonies rituelles au cours

\section{Au livre 5, Livre de la Kedusha.}

23. La shehita s'effectue en aval du larynx, en amont de la bifurcation de la trachée (Yoré Déa 20,1). L'incision doit se pratiquer au milieu du cou (Yoré Déa 20,3 ), et ne comprend que les parties molles. Les vertèbres cervicales ne doivent en aucun cas être touchées. Le Talmud (traité Houlin 28a) et le Choul'hane Aroukh (XXI, 1) nous indiquent les impératifs techniques de la shehita: incision de la plus grande partie de la trachée et de l'œsophage pour les mammifères ruminants et ongulés, incision de la plus grande partie de l'un de ces deux organes pour les oiseaux. 
desquelles des objets sont manipulés et agissent comme garants de la validité des actes ou des relations qu'ils accompagnent.

\section{Les mots et les choses: le serment more judaico}

C'est paradoxalement dans les sphères juridique et judiciaire, là où la fides, dans ses deux acceptions de foi et de confiance, est fondamentale, que les objets jouent un rôle important. Qu'ils soient rituels ou pas de prime abord, ils se trouvent investis d'un poids écrasant et font figure d'objets transitionnels, d'objets-liens, de fétiches ou talismans, en particulier dans le cadre des relations entre Juifs et Chrétiens. C'est le cas notamment de deux éléments, qui ne sont pas à proprement parler des objets mais qui agissent comme tels: ce que les documents appellent la «loi de Moïse $»^{24}$ incarnée dans divers supports, et le livre des Malédictions ${ }^{25}$. Dans l'espace aragonais, on a recours à ces deux objets lors de situations spécifiques: lorsqu'il faut garantir la validité de contrats conclus entre des Juifs et des Chrétiens, ou pour assurer la validité de la prestation de serments lorsque des personnes de l'une et l'autre confessions sont en contentieux. Les articles 138 et 139 des Fueros de Aragón ${ }^{26}$, promulgués au milieu du XIII ${ }^{\mathrm{e}}$ siècle par le roi Jacques $\mathrm{I}^{\mathrm{er}}$, officialisent le rituel et prévoient que dans le cas de contrats de prêts, la garantie (sous la forme du serment) dépend du montant considéré. L'article 138 débute par les mots suivants: «Ceci est le serment que les Juifs doivent prononcer en tenant la Torah». Quant à l'article 139, il débute ainsi: «Si tu sais la vérité et que tu jures faussement et que tu mens, alors viendront sur toi les malédictions

24. Il en est déjà question pour la période du Moyen Âge central. Michel Zimmermann, Écrire et lire en Catalogne $\left(I X^{e}-X I I^{e} s\right)$, Madrid, Casa de Velázquez, 2003, p. 1139, expose la forme du serment prononcé par les Juifs à Barcelone: «Il doit tenir la main levée sur sa Loi, et dire, par Dieu qui est apparu à Moïse dans le buisson ardent et qui a libéré les fils d'Israël d'Égypte et qui leur a fait traverser la mer Rouge à pied sec, $[\ldots]$ les choses suivantes». La formule figure dans un manuscrit de la Bibliothèque de Catalogne, ms. 1845, $\mathrm{f}^{\circ} 4$.

25. Il s'agit en fait de la longue suite de malédictions qui figurent en Deutéronome. 28. Celui qui jure sur le livre des Malédictions se condamne luimême et de manière automatique en cas de parjure, car il effectue une "autoimprécation» selon l'expression de Walter Pakter, Medieval Canon Law and the Jews, Ebelsbach, Rolf Gremer, 1988, p. 209.

26. Los Fueros de Aragón, según el manuscrito 458 de la Biblioteca nacional de Madrid, éd. Gunnar Tilander, Lund, C.W.K. Gleerup, 1937. 
suivantes $\gg^{27}$. Dans le premier cas, il est bel et bien fait mention d'un objet: la Torah que l'on tient, même si le plus souvent ce n'est pas le rouleau tout entier qui est manipulé; dans le second cas, c'est un chapitre de cette même Torah qui sert de garantie ${ }^{28}$.

De fait, la documentation fournit plusieurs témoignages de l'application réelle de cette procédure. Ainsi le roi Pierre III, fils de Jacques I ${ }^{\mathrm{er}}$, rappelle trente ans après la promulgation des Fueros, la procédure en vigueur dans une lettre adressée à la communauté juive d'Alagón ${ }^{29}$. Les Juifs ont le privilège - mais on a vu qu'il s'agissait aussi d'une garantie pour les Chrétiens - de prêter serment sur la loi de Moïse et non sur une charte.

Salomon ben Adret (dit aussi Rashba selon son acronyme), talmudiste et halakhiste, traite dans un de ses responsa (suite à une question posée par un correspondant) de la licéité de la prestation de serment sur des livres que les Chrétiens affirment être l'équivalent des dix commandements. Le sage recommande de faire attention, car il arrive que "les livres contenant les dix commandements soient les œuvres de religieux fanatiques et contiennent des propos idolâtriques $\gg^{30}$. Il préconise donc de s'assurer que les livres sur lesquels on peut être amené à jurer ne sont pas de cette sorte.

\section{Des rites profanes dans les fueros}

En dehors de ces exemples où l'objet est spécifiquement juif (la Torah ou la loi de Moïse), bien qu'annexé pour un rituel non-juif, la

27. Ibid. art. 138: «Aquesta es la jura que deuen iurar los iudios teniendo la tora en el cuello e deue dezir assi : "Tu iudio iuras por aquel qui dixo: io so e no y a otro nenguno sino yo" (Di) : "juro"». Art. 139: «Si sabes la verdat e iuras falsedat e mentira viengan sobre ti aquestas maldiciones e conprengan te (responde) "amen" ".

28. L'article 134 prévoit le cas où c'est un Chrétien qui prête serment. Très classiquement ce sont les évangiles qui tiennent lieu d'objet-garantie: «[...] el cristiano deve jurar por la cabeça de un cristiano e de VI dineros troa XII dineros por la cabeça de so padrino e de XII dineros en suso sobre libro e cruç [...]».

29. Archives de la Couronne d'Aragon (ACA dans la suite des notes), Chancellerie Royale, reg 39 f $135 \mathrm{v}$, Daroca 30.12.1276, Régné (réédité par Yom Tom Assis, History of the Jews in Aragon, Regesta and documents 1213-1327, Jerusalem, Magnes Press, 1978) $n^{\circ}$ 671: «Volumus etiam quod si tam vertatur inter vos et christianum [...] sit necessarium sacramentum, vos sacramentum non super cartam, set super legem Moysi illud tantum modo faciatis prout hac in quodam privilegio concesso [...] domine Jache felicis recordationis patre nostri ».

30. Responsum de Rashba $\mathrm{n}^{\circ} 218,3^{\mathrm{e}}$ partie, édité dans le CD-Rom Responsa Project. 
documentation fournit un cas de rituel qui paraît au moins en partie exogène. Le contexte est là encore juridique, puisque ce sont les coutumes de Tortosa ${ }^{31}$ qui évoquent dans un de leurs articles un rituel spécifique et garantissant les contrats conclus entre Juifs et Chrétiens. La rubrique 30 du livre 9 mentionne en effet: «Les juges approuvent la forme du serment des Juifs qui est décrite ainsi: "On place le Juif de la façon suivante, c'est-à-dire avec une Torah, une bassine d'eau et une chandelle allumée jusqu'à la fin" $\gg^{32}$. Si l'on retrouve le livre sacré des Juifs, la bassine d'eau et la chandelle ne sont mentionnées nulle part ailleurs. Il y a donc fusion d'héritages, syncrétisme culturel où des pratiques communes se voient associer des modalités tout à fait originales. Quant à la signification des objets ajoutés, elle nous reste inconnue, mais il me semble que cela ne constitue pas un point fondamental. Le plus important dans tout rituel, même hermétique, étant le(s) geste(s) accompli(s) et l(es)'objet(s) manipulé(s).

Étudions à présent le cas où des objets rituels des Juifs servent de catalyseur aux sentiments antijuifs dans un contexte de durcissement, au tournant des $\mathrm{XIII}^{\mathrm{e}}$-XIV ${ }^{\mathrm{e}}$ siècles.

\section{LES OBJETS ASSOCIÉS AUX RITES JUIFS, CIBLES ET MARQUEURS}

Alors que les objets rituels peuvent fédérer l'identité juive, $a$ contrario ils peuvent aussi cristalliser les sentiments antijuifs. C'est le cas en particulier d'un objet qui, au Moyen Âge, a donné lieu à de nombreux fantasmes chez les Chrétiens: le Talmud, moins en tant qu'objet stricto sensu il est vrai qu'en ce qu'il incarne de transgressif à leurs yeux. Très longtemps méconnu, il fait l'objet d'une campagne intense de dénonciation à partir du XII siècle au motif qu'il serait blasphématoire à l'encontre de Jésus-Christ ${ }^{33}$.

31. Les coutumes de Tortosa ont été mises par écrit en 1279 et trouvent leur origine dans les chartes de peuplement accordées par le comte Berenguer en 11481149 aux habitants de la ville. Elles ont été éditées par Jesus Massip Fonollosa, $L a$ gestacio de les costums de Tortosa, Tortosa, Consell Interterritorial de les Terres de l'Ebre, 1984.

32. Livre 9, rubrique 30: «Formam iuramenti iudeorum que inferius ponitur approbant arbitri preter id quod in ultimo paragrapho que incipit: Lo juheu com hom etcetera ponitu scilicet de thora, de bacino aque, et de candela incenssa que omnia et alia que sequntur usque ad finem repprobant ut iam supra in alio loco dixerunt».

33. Peter Schäfer, Jesus in the Talmud, Princeton-Oxford, Princeton University Press, 2007, p. 95 sqq. 


\section{Le Talmud, le rite et le blasphème}

Ce sont en réalité des Juifs convertis (Pierre Alphonse, Nicolas Donin, Paul Chrétien) qui, aux $\mathrm{XII}^{\mathrm{e}}-\mathrm{XIII}^{\mathrm{e}}$ siècles, informent les Chrétiens de l'importance du Talmud ${ }^{34}$. Consulté, étudié comme l'Ancien Testament, ouvrage saint des deux religions, le Talmud, même s'il n'est pas le Verbe, est extrêmement précieux, mais non saint et à ce titre il est une cible facile pour le polémiste. Mis en accusation au moment de la controverse de Paris en 1240, il est condamné à l'issue de la dispute et plusieurs centaines d'exemplaires sont brûlés à Paris ou à Vincennes (les sources divergent) ${ }^{35}$. Ouvrage de science et objet sacré, l'objet Talmud est devenu rituel par le rituel même qui le condamne, c'est-à-dire l'autodafé. Après Paris, où un second autodafé suit le premier deux ans plus tard, la fièvre anti-talmudique se répand et l'ouvrage est considéré avec suspicion un peu partout. Dans l'espace aragonais, la documentation de la pratique a conservé la mémoire de cette époque à travers les écrits royaux ordonnant la remise pour examen des exemplaires du Talmud aux ordres mendiants. En août 1263 s'est en effet tenue la dispute de Barcelone à l'issue de laquelle est proclamée la victoire du converti Paul Chrétien sur son adversaire le talmudiste Nahmanide, et le 29 août 1263 le roi d'Aragon s'adresse à ses sujets juifs. Son injonction est: «que vous envoyiez tous vos livres sous trois mois $»^{36}$. Mais, à la différence de Paris, les livres ne sont pas brûlés et semblent avoir été restitués à leurs propriétaires. En mars 1264, une lettre royale stipule en effet: «tous les blasphèmes contre notre Seigneur Jésus Christ et sa mère la sainte Vierge qui sont dans

34. Ainsi Gui Terré, qui n'est pas juif, explique dans sa question disputée (BnF ms. Lat. $16523 \mathrm{f}^{\circ} 83 \mathrm{r}$ ): «Utrum principalis articulus fidei nostre scilicet quod ponit trinitatem in unitate essencie possit probari contra iudeos per scripturas receptas ab eis?», en reprenant une phrase de Nicolas de Lyre à propos des sages du Talmud: «[...] les docteurs hébreux qui glosèrent l'Ancien Testament sont authentiques pour eux bien plus que pour nous, Jean, Augustin et les autres docteurs catholiques ».

35. André Tuilier, «La condamnation du Talmud par les maîtres universitaires parisiens, ses causes et ses conséquences politiques et idéologiques », Le brûlement du Talmud à Paris, dir. Gilbert Dahan, Paris, Le Cerf, 1999, p. 65, rappelle que «D'après les Extractiones de Talmut [...] la première crémation du Talmud dut intervenir en deux fois. Il y aurait eu quatorze charretées d'une part et six d'autre part».

36. ACA reg. $12 \mathrm{f}^{\circ} 111 \mathrm{v}, 29.08 .1263$. 
vos livres, vous devez les en retirer $»^{37}$. S'agissait-il d'exemplaires du Talmud ou pas, la question reste posée, mais dans tous les cas l'importance liturgique, sinon rituelle, de ces ouvrages est bien perçue alors qu' a contrario, le pouvoir (laïc ou ecclésiastique) ne ressent pas la nécessité de leur appliquer le cérémonial infamant et destructeur que constitue l'autodafé.

\section{Capes rondes et rouelles, des marqueurs}

Il nous faut évoquer également d'autres objets qui ne sont pas à proprement parler rituels, dans la mesure où ils ne sont pas constitutifs de rites spécifiques, mais qui sont tout à fait symboliques dans les relations entre Juifs et Chrétiens: les signes distinctifs, quelle que soit leur forme. On l'a dit plus haut, lorsque le port de signes distinctifs est prescrit par le $4^{\text {e }}$ concile de Latran en 1215, la forme de ces signes n'est pas précisée. Ce n'est que progressivement qu'apparaissent des dispositions précises. Dans l'espace qui nous occupe, le signe distinctif consiste en deux objets ou deux accessoires: la cape ronde et la rouelle. Coutume séculaire en péninsule Ibérique, héritage de pratiques musulmanes ${ }^{38}$, la cape ronde - similaire à une cape de pluie comme le précisent certains documents - est portée par les Juifs en Catalogne et, par extension, dans le royaume de Valence reconquis ${ }^{39}$. Une lettre pontificale adressée au roi d'Aragon en 1220 entérine la coutume du port de la cape ronde comme signe distinctif et dispense les Juifs de porter tout autre signe ${ }^{40}$. Ce n'est que plus tard que l'on voit se généraliser l'alternative entre cape ronde et signum, dans les années $1280^{41}$. On

37. ACA reg. 13 f 156 , Égéa 27.03.1264.

38. Les dhimmis portent en effet un habit distinctif dans les terres sous domination musulmane; les Chrétiens reconquérants récupèrent cette habitude.

39. L'analyse des Usatges de Barcelone ne montre pas trace de cette coutume, en revanche elle fait l'objet de l'article 23 des coutumes de Jaca, éd. José María Ramos y Loscertales, Barcelone, J. Bosch, 1927.

40. ASV Reg Vat. $11 \mathrm{f}^{\circ} 21 \mathrm{v}$, lettre du pape Honorius III à Jacques Ir en 1220, éd. Shlomo Simonsohn, The Apostolic See and the Jews. Documents 492-1404, Toronto, Pontifical Institute of Medieval studies, 1991, p. 111.

41. L'alternative se poursuit longtemps puisqu'on en trouve encore la trace en 1323, comme le montre l'exemple suivant: ACA reg $224 \mathrm{f}^{\circ} 49$, Barcelone 11.07.1323, Régné $\mathrm{n}^{\circ} 3272:$ : [...] iudei regnarum et terrarum nostrorum capis iudaicis non induuntur teneantur, in veste superiori rotulam panus coloris dissimile dicte vesti consueta portare». 
se rend compte d'une part que l'homogénéisation se fait lentement et, d'autre part, que les Juifs ressentent bien comme infamant le port de cette marque. Ils font tout pour en obtenir l'exemption à titre individuel ou collectif, temporaire ou définitif ${ }^{42}$. Mais peu à peu s'opère un durcissement et les exemptions ne sont plus données qu'au compte-gouttes. Dans le même temps, on observe que le signe fait l'objet de plus grandes précisions: en 1338, Pierre IV adresse une lettre à un de ses bailes et évoque un signum sive rotam. Quelque temps auparavant il était déjà question d'un rotulus in collo. Celui-ci doit être d'une couleur différente par rapport au vêtement, placée sur sa partie supérieure, et l'ensemble des membres de la communauté juive du lieu est soumis à cette obligation sans avoir le choix du signe. Il y a donc un nivellement par le bas puisque c'est le plus infamant qui est imposé à tous, la cape ronde étant en effet plus passe-partout. La lettre royale stipule enfin l'obligation faite au représentant du roi de sanctionner les manquements à ces prescriptions: «[...] il faut exiger absolument et percevoir une amende de la part de ceux qui contreviennent, comme cela a toujours été l'habitude dans la ville de Barcelone et dans les autres lieux de Catalogne dans lesquels demeurent des Juifs $\gg^{43}$. La règle est que tous doivent désormais porter un signe infamant marqueur d'une position d'infériorité sociale, et qu'il n'y a en principe aucun moyen de s'y soustraire. Rituelles ou pas, capes rondes et rouelles sont en tout cas indissociables du fait juif dans l'Occident chrétien de la fin du Moyen Âge.

\section{Des objets exogènes mais symboliques : les pierres de la lapidation pascale}

Venons-en à présent à un dernier cas, un peu extrême, peut-être critiquable mais qui mérite d'être évoqué, d'objets rendus rituels par l'usage que l'on en fait. Examinons donc le cas des pierres

42. En 1268, Jacques Ir $\mathrm{I}^{\text {er }}$ 'adresse à ses sujets juifs dans les termes suivants: «[...] nous vous concédons de ne pas porter la roue ou quelque autre signe sur la tête ou ailleurs sur votre corps », ACA reg $15 \mathrm{f}^{\circ} 123$, Cervera 25.10.1268, Régné $\mathrm{n}^{\circ}$ 392, publ. Francisco de Bofarull i Sans, «Jaime y los Judios», I Congreso d'Historia de la Corona de Aragon, Barcelone, 1913, p. 892, $\mathrm{n}^{\circ} 1 \mathrm{xxi}$.

43. ACA reg $595 \mathrm{f}^{\circ} 22 \mathrm{v}$, Barcelone 25.04.1338: «[...] a contrafacientibus exigenda penitus et levanda prout in civitate barchinone et aliis locis cathalonie in quibus commorantur iudei est fieri assuetum et hoc non mutetis». 
de la lapidation pascale, jetées rituellement lors de la semaine Sainte contre les habitations du quartier juif et leurs occupants. La détermination des pierres comme objets rituels est soumise à discussion dans la mesure où la pierre est sans doute l'arme la plus commune et la plus commode, celle que l'on trouve le plus facilement comme on en a encore parfois la preuve aujourd'hui. C'est l'arme du pauvre ou l'arme de circonstance. Pourtant, ce choix peut aussi se justifier dans la mesure où la pierre apparaît comme l'outil de prédilection pour les mises à mort dans l'Ancien Testament ${ }^{44}$. C'est par lapidation que sont exécutées les personnes condamnées pour avoir contrevenu aux injonctions divines. Ce n'est donc, me semble-t-il, pas un hasard si les émeutiers qui s'en prennent aux Juifs lors des cérémonies commémorant la Passion du Christ (processions, messes) choisissent la lapidation comme modus operandi. Il s'agit évidemment, si l'on retient cet exemple comme valide, d'objets exogènes qui sont inclus dans un rite spécifique, un rite d'exclusion. Il faut rappeler en effet que jusqu'assez tard, fin XIII-début XIV ${ }^{e}$ siècle, c'est la «convivence» qui domine dans les relations entre Juifs et Chrétiens, en péninsule Ibérique tout du moins. Les nombreuses dispositions canoniques adoptées par l'Église, qui insiste de manière récurrente et parfois chaque année, sur la séparation nécessaire entre membres de confessions différentes, montrent a contrario que c'est la mixité qui domine. En ce sens, les violences pascales sont très certainement des violences rituelles, destinées à rappeler symboliquement, une fois par an, qu'il existe des limites et qu'il ne faut pas les dépasser. Les pierres utilisées alors, par des enfants et des petits clercs le plus souvent ${ }^{45}$,

44. Les exemples abondent en effet, où la lapidation est mentionnée explicitement comme mode de mise à mort: en Lv. 20. 2, pour condamner quiconque aurait sacrifié son enfant au culte de Moloch et, plus tard, en Nb 15. 35-36 (le verset précédant celui prescrivant le port des franges rituelles) pour condamner un homme ayant transgressé le shabbat: "Alors l'Éternel dit à Moïse : "Cet homme doit être mis à mort; que toute la communauté le lapide hors du camp". Et toute la communauté l'emmena hors du camp et on le fit mourir à coups de pierres, comme l'Éternel l'avait ordonné à Moïse».

45. Voir l'exemple de Gérone: en 1327, Alphonse IV s'adresse à l'évêque de Gérone dans les termes suivants: «[...] le jeudi et le vendredi Saints avant la fête de la Résurrection de notre Seigneur, des clercs et des membres de votre familia, ont agressé et provoqué des dommages [...]», ACA reg 428, f124, Barcelone, 25.01.1327. Et encore: ACA Reg 623 f $^{\circ} 6$, Barcelone 7 mars 1342, à propos des événements qui se sont déroulés à Villafranca : « $[. .$.$] pueri aliqui vagabundi contra$ iudeos lapidare ac eis inferre verbo vel facto in personis et bonis $[\ldots] »$. 
sont les objets, rituels, de ce rappel. Elles permettent l'affirmation de la nécessité de l'exclusion, le rappel de la séparation, mais elles sont jetées sur les maisons (et non sur les personnes), dont les habitants doivent rester chez eux, portes et volets fermés. Elles assurent le bruit et la publicité du rite, la visibilité ou la sonorité de la performance, tout en limitant les risques réels d'homicide. D'ailleurs les autorités laïques tout comme les responsables communautaires juifs anticipent souvent les violences et font tout pour s'en prémunir ${ }^{46}$. Parfois cependant, le rite dérape et la démonstration tourne au massacre, comme à Gérone en 1293 ou à Lérida en 1333, où des Juifs ont été mortellement blessés parce qu'en plus des jets de pierres, les assaillants ont incendié les maisons à l'aide de torches ${ }^{47}$ ou utilisé des balistes pour projeter des pierres de grosse taille. On voit alors le pouvoir s'exprimer pour dénoncer les excès et appeler au châtiment des responsables ${ }^{48}$. Mais le plus souvent, la lapidation pascale reste encadrée dans son déroulement habituel. Les pierres agissent bien dans ces moments de rappel de la norme, comme objets rituels, garants d'une situation connue et presque encadrée.

\section{Conclusion}

On a choisi ici de retenir une définition très large de l'objet rituel, une définition non orthodoxe, quitte parfois à sembler construire cette définition. Ont ainsi été considérés des objets qui, parce qu'ils étaient manipulés ou utilisés dans un cadre rituel, ont été considérés comme objets rituels. C'est donc le rite, qu'il soit liturgique ou non, qui fait pour nous l'objet rituel. Ce parti pris, non exclusif, nous a permis en effet d'englober dans l'analyse un éventail

46. Ainsi en 1268, le roi prévoit à Játiva que: «[...] personne ne puisse jeter des pierres dans les maisons de ces Juifs et que vous fassiez stationner un homme à la porte Algefne le jour du Vendredi saint chaque année après la messe », ACA reg $15 \mathrm{f}^{\circ} 95 \mathrm{v}$, Valence, 26.04.1268, Régné $\mathrm{n}^{\circ} 377$.

47. ACA reg $87 \mathrm{f}^{\circ} 60 \mathrm{v}$, Barcelone, 7.04.1293: «[...] invaserunt aliquos domos ipsorum iudeorum prohiciendo ignem in ipsis domibus [...] depredaverunt [...] et vulnerando etiam aliquos iudeos cum ballista in domibus suis $[\ldots] »$.

48. La documentation royale rapporte en ces termes les événements de Lérida en $1333:$ «[...] ac quod deterius est impie et improvide letaliter sauciantur», ACA reg $471 \mathrm{f}^{\circ} 96 \mathrm{v}$, Valence 11.03.1334. 
d'objets et, partant, un éventail de situations qui nous ont fait saisir la complexité et la richesse de la coexistence d'une minorité religieuse, la minorité juive, au sein de la majorité chrétienne, à un moment de tension voire de retournement de conjoncture. La documentation très riche dans l'espace méditerranéen à la fin du Moyen Âge et les textes hébraïques et latins comme l'iconographie fournissent des exemples nombreux. Il est intéressant toutefois de voir que les objets et en particulier les objets rituels, endogènes ou exogènes, sui generis ou plaqués, peuvent servir, au-delà ou en plus des textes, à exprimer toutes sortes de relations, à médiatiser les passions ou symboliser les différences. L'objet rituel dans ce contexte, loin d'être seulement religieux ou cultuel, est donc aussi pleinement social et politique.

claire.soussen@wanadoo.fr 